INPLASY

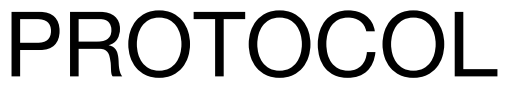

To cite: Cai et al. Effectiveness and Safety of different dressings therapy for pressure injuries: a protocol for systematic reviews and network meta-analysis. Inplasy protocol 2020100087. doi: 10.37766/inplasy2020.10.0087

Received: 22 October 2020

Published: 23 October 2020

Corresponding author:

Yitong Cai

caiytlucky@163.com

Author Affiliation:

Lanzhou University

Support: 17JR5RA235.

Review Stage at time of this submission: Data extraction.

Conflicts of interest:

The authors have no conflicts of interest to disclose.

\section{Effectiveness and Safety of different dressings therapy for pressure injuries: a protocol for systematic reviews and network meta-analysis}

Cai, YY1; Zhou, YY2; Xing, LN3; Kang, YY4; Li, HL5; Cheng, P6; Wang, YJ7.

Review question / Objective: For pressure ulcer patients, which dressing is the safest and most effective.

Condition being studied: Pressure injuries, also known as pressure ulcers, are local skin injuries. Once a pressure injury occurs, clinical treatment is relatively difficult, the treatment cycle is long, and the treatment cost is high, which brings a heavy burden to patients and society. Therefore, look for reliable pressure injuries treatment method is one of the focus of clinical nursing workers

INPLASY registration number: This protocol was registered with the International Platform of Registered Systematic Review and Meta-Analysis Protocols (INPLASY) on 23 October 2020 and was last updated on 23 October 2020 (registration number INPLASY2020100087).

\section{INTRODUCTION}

Review question / Objective: For pressure ulcer patients, which dressing is the safest and most effective.

Rationale: At present, there are many kinds of dressings for the treatment of pressure injuries, and there is no uniform conclusion about which dressing is the most effective. Therefore, we systematically evaluate the effects of different dressings on the treatment of pressure injuries.

Condition being studied: Pressure injuries, also known as pressure ulcers, are local skin injuries. Once a pressure injury occurs, clinical treatment is relatively difficult, the treatment cycle is long, and the treatment cost is high, which brings a heavy burden to patients and society. Therefore, look for reliable pressure injuries 
treatment method is one of the focus of clinical nursing workers

\section{METHODS}

Search strategy: \#1 "Pressure Ulcer"[Mesh] \#2 pressure ulcer*[Title/Abstract] OR bedsore*[Title/Abstract] OR pressure sore*[Title/Abstract] OR bed sore*[Title/ Abstract] OR decubitus ulcer*[Title/ Abstract] OR decubital ulcer*[Title/ Abstract] OR decubitus ulceration[Title/ Abstract] OR decubitus ulcer*[Title/ Abstract] OR ulcers decubitus[Title/ Abstract]) \#3 \#1 OR \#2 \#4 "Randomized Controlled Trials as Topic"[Mesh] \#5 randomized controlled trial [Publication Type] OR random*[Title/Abstract] \#6 \#4 OR \#5 \#7 "Bandages"[Mesh] \#8 "Bandages, Hydrocolloid"[Mesh] \#9 "Occlusive Dressings"[Mesh] \#10 "Honey"[Mesh] \#11 "Hydrogels"[Mesh] \#12 "Alginates"[Mesh] \# 13 "Negative-Pressure Wound Therapy"[Mesh \#14"Silver"[Mesh] \#15"Silver Sulfadiazine"[Mesh] \#16 "Collagenases"[Mesh] \#17 Bandage*[Title/ Abstract] OR dressing*[Title/Abstract] OR gauze[Title/Abstract] OR tulle[Title/ Abstract] OR film*[Title/Abstract] OR bead[Title/Abstract] OR Pad*[Title/ Abstract] OR foam*[Title/Abstract] OR hydrocolloid*[Title/Abstract] OR "sodium hyaluronate" [Title/Abstract] OR alginat ${ }^{\star}\left[\right.$ Title/Abstract] OR hydrogel ${ }^{\star}[$ Title/ Abstract] OR silver*[Title/Abstract] OR honey*[Title/Abstract] OR Foam*[Title/ Abstract] OR non-adherent[Title/Abstract] OR "non adherent"[Title/Abstract] OR matrix [ Title/Abstract] OR Collagenase*[Title/Abstract] \#18 \#7 OR \#8 OR \#9 OR \#10 OR \#11 OR \#12 OR \#13 OR \#14 OR \#15 OR \#16 OR \#17 \#19 \#3 AND \#6 AND \#18.

Participant or population: Patients of any age described as having PIs. Studies were excluded if the study included other types of wounds (such as chronic wounds and venous leg ulcers) or if the subjects were animals.

Intervention: Hydrocolloid dressings, silver dressings, foam dressings, saline gauze, petrolatum gauze, collagen dressings, danghui dressings, honey dressings and other dressings or conventional treatment.

Comparator: Other dressings or conventional treatment.

Study designs to be included: Randomized Controlled Trial (RCT) published in Chinese or English language without restriction on the use of blind methods.

Eligibility criteria: The RCTS were for patients with pressure ulcers and the interventions were dressings or traditional treatments.

Information sources: We will search the following English electronic bibliographic databases: PubMed (inception- present), Embase (inception- present), Cochrane Central Register of Controlled Trials (CENTRAL) (inception- present), CINAHL (inception- present), Web of Science (inception- present), as well as the Chinese databases: China Knowledge Network (CNKI) (inception- present), China Biomedical Literature Database(CBM) (inception- present), VIP Data(inceptionpresent), Wan Fang Data(inceptionpresent). Furthermore, reference lists of included RCTs and relevant systematic reviews will be searched. There will be no restrictions on publication year.

Main outcome(s): (1) Effectiveness: Time to complete healing/rate of healing; (2) Safety: wound infection, bacteria amount, pain during treatment; (3) Cost.

Additional outcome(s): Length of hospital stays, incidence of different type of infection.

Quality assessment / Risk of bias analysis: The methodological quality of the final included RCT will be evaluated independently by two reviewers (HLL, PC). Any disagreements will be resolved through discussion between the two parties or decided by a third reviewer (YJW). Evaluate the quality of the literature according to the recommended bias risk assessment tool Cochrane 5.1.0. The evaluation contents include: random 
sequence generation, allocation concealment, blind method of participants, researchers and result evaluators, integrity of outcome indicators, selective reporting, other source bias, etc. Each was rated as "high risk of bias," "unclear," and "low risk of bias.

Strategy of data synthesis: R 3.5.0 Software gemtc package and JAGS 3.4 .0 software were used for data analysis, and Stata $\mathbf{1 5 . 0}$ was used to draw the network diagram and funnel diagram. We will calculate the mean differences (MDs) or standardized mean differences (SMDs) with 95\% confidence interval $(95 \% \mathrm{Cl})$ for continuous variable data, and relative risk (RR) with $95 \% \mathrm{Cl}$ for dichotomous variable data. Set the number of pre-iterations to 10,000 and the number of iteration operations to 100,000 . The statistical heterogeneity will be examined using the 12 statistic and $P$ value. 12 was used to judge the size of heterogeneity, 12 $\leq 50 \%$, it can be considered that the homogeneity among studies is good; If 12 $>50 \%$, it is considered that the heterogeneity among studies is large, and multiple regression model is adopted for processing.

Sensibility analysis: If the evidence is sufficient, we will conduct a subgroup analysis to determine the difference between different gender, age ((Over 60 years old, less than 60 years old, different stages of PIs, courtiers) etc.

Country(ies) involved: China.

Keywords: Pressure injuries, network meta-analysis, dressing.

Contributions of each author:

Author 1 - Yitong Cai - Conceptualization, Methodology. Software, Writing - original draft, Writing - review and editing.

Author 2 - Yuying Zhou - Conceptualization.

Author 3 - Lina Xing - Methodology.

Author 4 - Yingying Kang - Methodology.

Author 5 - Hailing Li - Software.

Author 6 - Peng Cheng - Writing - original draft.

Author 7 - Yujuan Wang - Writing - review and editing. 